\title{
Threat Assessment Teams for Institutions of Higher Education: A Review of Key Ideas and Practices for Professional Counselors
}

\author{
Rebecca Bolante $^{1}$ (D) https://orcid.org/0000-0003-3028-9354 \\ Cass Dykeman ${ }^{2}$ (D) https://orcid.org/0000-0001-7708-1409 \\ ${ }^{1}$ Bolante.net \\ ${ }^{2}$ Oregon State University
}

\begin{abstract}
This is the author's preprint. The copy of record was published as the following:

Bolante, R., \& Dykeman, C. (2017). Threat Assessment Teams for Institutions of Higher Education: A Review of Key Ideas and Practices for Professional Counselors. Vistas Online 2017, Article 29.

https://www.counseling.org/docs/defaultsource/vistas/Threat Assessment Teams.pdf?sfvrsn=10c24a2c 4
\end{abstract}

\begin{abstract}
This review of literature provides a comprehensive account of the instigative factors, history, and evolution of threat assessment teams (TATs) for target-based violence in institutions of higher education (IHEs). Through examining diverse approaches to threat assessment, this review investigates the most effective criteria for creating protocols to identify and manage threats of target-based violence. The objective is to provide a greater understanding of the precursors and warning signs to threats of violence, including an understanding of what psychosocial factors impel students to enact mass murder, so that TATs can implement preventative strategies to school violence. The generalized findings of the majority of studies point to the necessity for a multi-disciplinary team referencing fact-based predictors of violence, yet applying an individualized and preventative approach to each case of suspected violence.
\end{abstract}

Keywords: threat assessment teams, school violence, target-based violence

\section{Introduction}

Physical and psychological safety are essential aspects of a healthy learning and working environment and can affect retention and completion rates for higher education. As such, the development and implementation of a threat assessment team (TAT) is a vital aspect of ensuring safety in institutions of higher education (IHEs). The rationale for exploring the topic of threat assessment in schools is to save lives through developing a comprehensive understanding of the best 
techniques and strategies for identifying and managing threats of violence. TATs are a newer phenomenon in the academic landscape, and professional counselors may not be familiar with specialized TAT terms. As such, definitions of TAT key terms will be presented before turning to an exploration of the rationale and design for this review.

\section{Glossary of Specialized TAT Terms}

Threat. An expression of intent to do harm or act out violently against someone or something (including self; University of Arkansas at Little Rock Public Safety Department, 2017a). Specifically, threats "can be spoken, written, or symbolic. Not all threats, nor those that threaten, are equal in their potential for harm, but all threats that are brought to the attention of the university should be taken seriously, evaluated, and documented" (University of Arkansas at Little Rock Public Safety Department, para. 1). Threats can be classified in four types: (a) direct, (b) indirect, (c) veiled, and (d) conditional (University of Arkansas at Little Rock Public Safety Department, 2017b).

Direct threat. This threat can be defined as: "a specific act against a specific target and is delivered in a straightforward, clear, and explicit manner - 'I am going to place a bomb in the school's gym"' (Indiana Department of Education, Safety Academy, 2017, p. 1).

Indirect threat. This threat can be defined as one that: Tends to be vague, unclear, or ambiguous. The plan, the intended victim, the motivation, and other aspects of the threat are masked or equivocal: 'If I wanted to, I could kill everyone at this school'. Violence is implied but tentatively 'if I wanted to' and suggests a violent act COULD occur not that it WILL occur. (Indiana Department of Education, Safety Academy, 2017, p. 1).

Veiled threat. This threat can be defined as one that: "Strongly implies but does not explicitly threaten violence. 'We would be better off without you around anymore' clearly hints at a possible violent act, but leaves it to the potential victim to interpret the message and give a definite meaning" (Indiana Department of Education, Safety Academy, 2017, p. 1).

Conditional threat. This threat can be defined as one that: "Warns that a violent act will happen unless certain demands or terms are met: 'If you don't pay me one million dollars, I will place a bomb in the school'" (Indiana Department of Education, Safety Academy, 2017, p. 1).

Threat Assessment Team. A group of officials that convene to identify, evaluate, and address threats or potential threats to school security (U.S. Department of Education, 2017). In particular, these teams “. . . review incidents of threatening behavior by students (current and former), parents, school employees, or other individuals ... Some schools may need assistance in 
determining whether a health or safety emergency exists in order to know whether a disclosure may be made under FERPA's health or safety emergency provision. Accordingly, members of a threat assessment team might include officials who can assist in making such decisions, such as school principals, counselors, school law enforcement unit officials, as well as outside medical and mental health professionals and local law enforcement officers" (U.S. Department of Education, 2017, p. 1).

\section{Rationale for the Present Study}

Threat assessment is a young scientific discipline (Meloy \& Genzman, 2016). As such, there is a need in the counseling field for better understanding of the concept of threat assessment and management to enhance campus safety within the parameters of the counselor's ethical and legal considerations. One dilemma with which counselors continue to struggle is maintaining safety while balancing confidentiality and privacy. This can be especially challenging when considering the additional layers of disability laws and documentation. Threat assessment and management best practices are very new for IHEs and for counseling professionals. The area of counseling within IHEs can be complex and varied. Counselors can have obligations that range from academic advising, personal counseling, and crisis intervention, to trauma response. Each institution has its unique culture of how counselors' functions are structured and implemented. Some colleges embrace the need for support and care for students through mental health counseling, while others focus on counseling related to academic advising. With their clinical expertise as well as their connections with community resources, the role of the counselor is instrumental for campus TATs (Randazzo \& Cameron, 2012). Nonetheless, threat assessment within IHEs has a tremendous impact on the counselors within a college institution.

Both formal and informal training for counselors involves a high standard for student/client privacy and confidentiality. This is instilled through the various ethical codes enforced by the different licenses and certifications required by counseling entities. Perhaps more than other TAT members, counselors must also be aware of their personal and professional ethics, in addition to the state and federal regulations around information sharing. Because there is such a broad sense of confusion around information sharing regarding when to share, how much information to share, and the importance of sharing information (which can be vital for mitigating situations of concern), some states have initiated legislation for clarification. For example, Virginia state laws pertaining to threat assessment provide guidance through model policy, procedures, and guidelines for assessing threats and were developed in response to legislation enacted by the Virginia 
General Assembly in 2013 (Perloe \& Pollard, 2016; Virginia Department of Criminal Justice Services 2014).

\section{Plan for this Study}

This systematic review of literature has two objectives. These are to: (1) summarize best practices and recommendations for TATs, and (2) indicate the unique needs for threat assessment and management for IHEs. A primary goal of the present study is to assess the literature and to determine the essential questions used when assessing and managing a threat situation at IHEs. Results are envisioned to inform TAT professionals of potential training implications for best practices. The findings contribute to the process of developing and training TAT professionals within IHEs. Results will also provide new findings for future employees in colleges and universities relative to their differing needs for threat assessment.

\section{Design}

\section{Method}

This study employed a systematic review (without meta-analysis) design (Moher, Liberati, Tetzlaff, Altman, \& PRISMA Group, 2009). The authors used the procedures for this design that were set forth in the Preferred Reporting Items for Systematic Reviews and Meta-Analyses (PRISMA; Moher et al., 2009) The PRISMA Statement consists of a 27-item checklist of items to include when reporting a systematic review. The aim in using PRISMA is to maintain consistency among research; to include both systematic reviews and meta-analysis; and to allow the research project to be duplicated by others (Moher et al., 2009). The authors used the PRISMA Flow Diagram to structure the systematic review (PRISMA, 2017). This design was selected since it represents the gold-standard for such reviews (Ottawa Hospital Research Institute, 2009).

\section{Inclusion Criteria}

The article inclusion criteria for this study was fourfold. These four criteria were: (1) published in English, (2) fits a pre-set publication time frame, (3) available full-text online, (4) included a TAT factor (i.e., prevention of targeted acts of violence, warning behavior signs, threat assessment, threat management, and best practices for violence prevention. See Table 1.

\section{Information Sources}

The following electronic databases were used in this search: EBSCOhost, PsycINFO, and HSDL (Homeland Security Digital Library). The titles and 
abstracts of publications identified through these searches were reviewed, and their relevance to the research topic was determined. If an abstract indicated

Table 1

Inclusion and Exclusion Criteria

\begin{tabular}{ll}
\hline \multicolumn{1}{c}{ Inclusion criteria } & \multicolumn{1}{c}{ Exclusion criteria } \\
\hline Focus on IHE & Environmental threats \\
Threat assessment & Testing (diagnostic) \\
Violence & Science \\
Shooting & Workplace violence \\
English language & Non-English language \\
Published during 1990-2012 & \\
Scholarly, peer reviewed & \\
Qualitative and quantitative & \\
\hline
\end{tabular}

that a publication was relevant, a full transcript of the paper was obtained. In addition to searching the aforementioned databases, books and Web sites of relevant associations were searched to identify other possible sources of information. The following programs were contacted and queried about new research being conducted on Threat Assessment in IHEs by leading authors in the field: Willamette Valley Adult Threat Advisory Team, Mid-Valley Student Threat Assessment Team, Association of Threat Assessment Professionals, Chemeketa Community College Threat Assessment Team, FBI Behavioral Analysis Unit, International Association of Campus Law Enforcement Administrators (IACLEA), University of Nebraska Public Policy Center, Factor One, Virginia Polytechnic Institute and State University, Bill \& Melinda Gates Foundation, and the University of Virginia. Finally, the Web of Science was accessed to identify cited sources from lead authors in the field of threat assessment. In particular, the work of the following authors was traced: Gene Deisinger, Reid Meloy, Mary Ellen O'Toole, Dewey Cornell, and Marisa Randazzo. These authors were selected because they either developed recommendations for TATs in IHEs or were regularly cited by other authors writing about TATs in IHEs.

\section{Keyword Search Strategy}

The following topics related to the research questions were investigated: threat assessment and management, higher education, campus violence, and prevention of targeted acts of violence. The following combinations of terms were used in database searches: college AND threat assessment NOT testing NOT diagnostic NOT aptitude NOT hazards NOT science NOT children mass murder AND schools AND multiple homicides. Hand searching of reference lists of included 
reviews was also conducted. If an abstract indicated that a publication was relevant, a full transcript of the paper was obtained.

\section{Outcome of Search Processes}

Figure 1 summarizes the results from each search stage (i.e., article identification to final article inclusion.

\section{PRISMA Fidelity}

Fidelity to the review PRISMA review standards was assessed using the PRISMA checklist (Moher et al., 2009). This included an itemized checklist focused on the subject/topic as a way to validate the quality of the search.

\section{Data Items}

The data items were defined by using the Population Intervention Comparison Outcome Study Design (PICOS, Higgins \& Green, 2011). This allowed for an extensive itemization of the variables for which the data were sought.

Population. The population included in this review included factors such as adult age, male and female, and higher education. Given the aforenoted youth of the threat assessment field, the search criteria encompassed a broader scope (such as youth and other locations).

Intervention. Based on the review, various techniques and strategies are being conducted depending on the variables related to the situation. These interventions range from actuarial tables to informed investigative practices. Some of the terminology for the interventions includes: threat assessment; threat assessment management; safety plans; risk Management; and risk assessment. These interventions also include a strong element of connecting with law enforcement.

Comparison. There are alternatives to the interventions that include elements of TATs but have unique and different approaches. Some of these alternatives are: behavioral assessment; profiling; and student/employee codes of conduct.

Outcomes. The desired or expected outcome of this systematic review is to gain clarification on best practices for TATs in IHEs. This would include improving confidence within the TAT teams in IHEs and, at the same time, reducing the incidence of targeted violence within the school campus and community. 


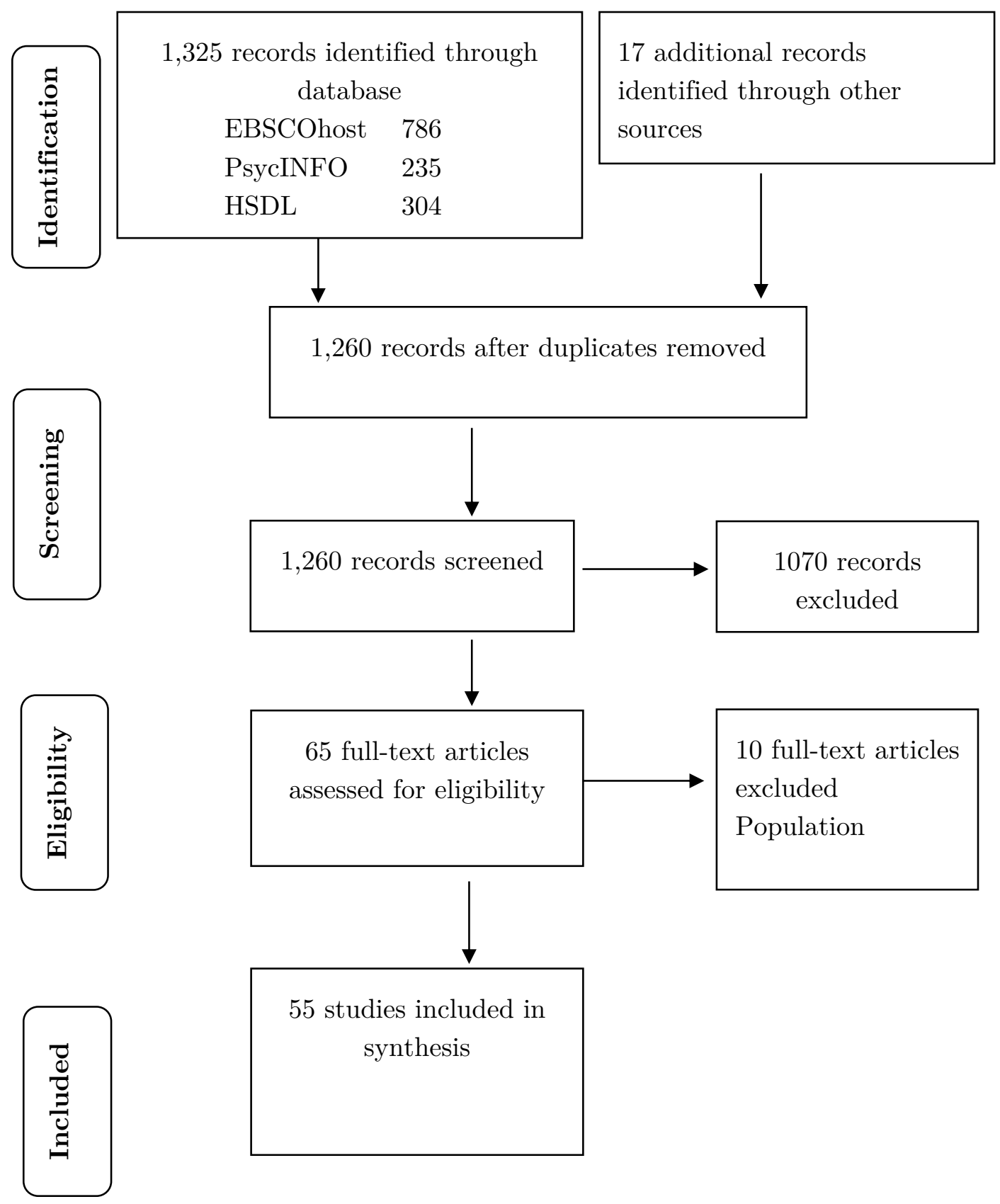

Figure 1. PRISMA flow diagram of the literature reviewing process. 
Outcome of results. The relevant and desired outcomes of this systematic review are all related to the prevention of targeted violence and providing the necessary support of students/people in need of interventions.

Study design. The randomized controlled group design represents the "gold standard" of outcome research since it controls for so many threats to internal validity (US Department of Education, 2003). However, in threat assessment research, this approach is not always possible for ethical reasons. Therefore, the study designs are based on retrospective data from past incidents. Although randomized controlled studies would provide a design with the least biased form of evidence, randomized controlled studies are not realistic within the intent of this study. The systematic review of literature included all study designs within the scope of exclusionary and inclusionary searches.

\section{Risk of Bias Across Studies}

Awareness of bias within studies and publications was taken into consideration. Few studies included this awareness of bias and the addressing of the potential conflicts. Conflicts identified included funding sources for the study, training materials, and content supporting the research. Some studies and publications focused on specific actuarial tools developed by the author to support the instrument's validity. Few publications clearly stated any bias or conflict of interest.

\section{Results}

The literature contained in the 55 articles involved in the synthesis were organized on the basis of four superordinate themes. These themes were: (a) origins, (b) targeted violence, (c) leakage, risk factors and warning behaviors, and (d) K12/IHE discontinuities.

\section{Theme 1: Origins of the Threat Assessment Team}

Overview. The TAT is born out of a major and comprehensive endeavor to achieve greater understanding of school threats and to generate strategies, trainings, and dynamic and interactive efforts by school, community, and law enforcement officials to identify and prevent school shootings (Albrecht, 2010; Cornell \& Allen, 2011; Cornell, Sheras, Gregory, \& Fan, 2009; Nolan, Randazzo, \& Deisinger, 2011; Scalora, Simons, \& VanSlyke, 2010). In fact, school violence was well-recognized by the U.S. government before the Columbine shootings. In 1998, Attorney General Janet Reno and Secretary of Education Richard W. Riley sent out a letter to U.S. schools calling for a "comprehensive violence prevention plan," 
which would become a cursory aspect of the entire U.S. school system (O'Toole, 2000, p. 5). A few years later, the National Center for the Analysis of Violent Crime constructed a threat assessment intervention model that describes "a methodical procedure for evaluating a threat and the person making the threat, with the aim of reaching an informed judgment on the danger that a violent act will actually be carried out" (O'Toole, 2000, p. 6).

In 1999, the Safe School Initiative (SSI) was developed through a collaborative effort by the U.S. Secret Service and the U.S. Department of Education (Pollack, Modzeleski, \& Rooney, 2008). By reviewing past incidents of targeted violence in schools, the SSI sought to identify repeating patterns and "whether pre-attack behaviors of perpetrators could be identified to prevent future attacks" (Pollack et al., 2008, p. 3). The SSI determined that in many cases of target-based violence in schools, the subject had either directly or indirectly revealed his/her intentions to others. In most cases, witnesses of such information said and did nothing to prevent the attack. Therefore, the SSI focused on educating students and school employees to identify and report such information to the appropriate authorities so as to better prevent future attacks.

The Willamette Valley Adult Threat Advisory Team, Chemeketa Community College Threat Assessment Team, and the Mid-Valley Student Threat Assessment Team in Salem, Oregon, are good examples of multidisciplinary teams that include a diverse membership of community professionals to approach the problem of target-based violence in schools (Van Dreal, Cunningham, \& Nishioka, 2005). These Salem threat assessment teams accomplish this task through identifying and addressing warning behaviors or concerning situations so that risk factors to the school community are reduced and protective factors are increased (Bolante \& Dykeman, 2015).

Higher education. The presence of violence in the U.S. education system also exists in IHEs, such as colleges and universities (Bondü, Cornell, \& Scheithauer, 2011; Cornell, 2010, 2011; Cornell et al., 2009). In 2007, an armed student at Virginia Tech shot and killed 32 people and wounded 23, before killing himself (Cornell et al., 2009). This tragic event spurred the college to create its own TAT. More recently, the tragic event in Roseburg, Oregon, at Umpqua Community College left 10 dead, including the shooter, and nine injured (Tuttle, 2015).

The need for a TAT in all campus environments is overwhelmingly apparent. The development of plans and protocols for the effective functioning of a TAT, therefore, is a high priority of the U.S. education system, law enforcement, and the United States as a country. This review of literature strives to provide an 
overview of progress towards creating comprehensive threat assessment teams and protocols throughout the U.S. education system.

\section{Theme 2: Threat Assessment Approaches for Targeted Violence}

Overview. Target-based violence in the form of mass killings is an absolute tragedy which demands that we work diligently to better understand and manage the problem. This review of literature hopes to provide inspiration and a broader knowledge base to the challenge of addressing and reducing violence in our schools.

Threat assessment focuses on targeted violence aimed at a particular person, group, or cause and is also known as target-based violence (Reddy et al., 2001). Meloy, Hoffmann, Guldimann, and James (2012) took care to distinguish threat assessment and risk assessment. They stated that: "Threat assessment is concerned almost wholly with the risk of targeted violence by a subject of concern, and has a behavioral and observational policing focus. Risk assessment may address different domains of risk than threat assessment, and typically relies on more historical and dispositional (status) variables" (p. 256).

Historically, there have been four main approaches to assess situations for the risk of target-based acts of violence: (1) unstructured clinical judgment, (2) profiling, (3) the use of automated or actuarial tools, including artificial intelligence and computerized databases, and (4) structured professional judgment (Borum, Cornell, Modzeleski, \& Jimerson, 2010; Guy, Packer, \& Warnken, 2012). Approaches number one, three, and four have also been correlated, respectively, as first-, second-, and third-generation threat assessment responses to the occurrence of violence (Bernes \& Bardick, 2007). As such, the four approaches mark an evolution in the formulation of strategies to identify and prevent violence in the form of mass murder at schools and in the public.

Unstructured clinical judgment. This approach for assessing a threat of targeted violence comprised an initial response to the problem by an official, probably a school counselor, who made an individual attempt to understand and resolve the situation. However, this approach lacked reference to an empirical body of knowledge and research into the topic of threat assessment risk factors or documented behavior warning signs (Bernes \& Bardick, 2007). Bernes and Bardick noted that "Such informal assessments may result in the over-identification of students at risk for committing a violent act" (p. 420). Therefore, without an informed background on the topic, unstructured clinical judgment was found to be very ineffective in its propensity to predict or intervene in school violence (Bernes \& Bardick).

Profiling. Profiling was one of the first somewhat structured attempts to compose a composite portrait of the violent perpetrator - how the person looked and behaved, the way the person thought, and their particular background (Borum 
et al., 2010; O'Toole \& Häkkänen-Nyholm, 2012; Sulkowski \& Lazarus, 2011). The idea implicit in profiling was that through analyzing actual violent perpetrators, potential perpetrators of violence could be more easily identified, then interventions could be made to prevent future violent acts from occurring (Cornell, 2011). Profiling is seemingly an attempt to resolve a very complex situation using an oversimplified method of reasoning (O’Toole \& Häkkänen-Nyholm, 2012).

Like unstructured clinical judgment, profiling has proven to be ineffective and to elicit an extremely high number of false-positive assessments for persons considered to be potentially violent (Borum et al., 2010). According to Vossekuil, Fein, Reddy, Borum, and Modzeleski (2004), both the U.S. Department of Education and the U.S. Secret Service reviewed target-based shooting incidents and concurred that "no accurate or useful demographic or social profile of school attackers" truly existed (Borum et al., 2010). Randazzo and Cameron (2012) categorized profiling not as a bona fide form of fact-based, deductive threat assessment, but rather as a form of generalized risk assessment.

Automated or actuarial tools. The development and use of actuarial tools in the assessment of threat for targeted violence was the first approach that utilized empirical risk factor and warning behavior data in order to better understand the degree of threat (Reddy et al., 2001). However, in the case of school shootings, due to the lack of relevant knowledge regarding the variables involved and the lack of frequency of the event, it has been impossible to arrive at statistically valid results using actuarial tools to assess the level of threat or likelihood of violence (Reddy et al., 2001). Of course, the hope is to increase knowledge of the variables while also preventing further violent incidents. Therefore, the use of actuarial tools to identify and assess threat and to prevent violence is not a significant solution to the problem.

Structured professional judgment. Structured professional judgment, also known as structured clinical assessment and guided professional judgment, is the umbrella term used for the most recent attempts to assess the threat of targetbased violence (Reddy et al., 2001, p. 2). Reddy et al. (2001) defined structured professional judgment as "a set of operational activities that combine the use of an investigative process and information-gathering strategies with target-violence relevant questions" (p. 6). The format of structured professional judgment entails an analysis of the risk factors and warnings behaviors exhibited by the subject of concern that are associated with actual known cases of violence (Reddy et al., 2001).

Unlike the previous three approaches to threat assessment, structured professional judgment involves a collection of evidence-based knowledge and deductive protocols for identifying and preventing violence. However, while based on empirical research, structured professional judgment also envisions threats of 
violence as highly dynamic, fluid, and ever-changing situations that must be continuously managed in an equally time and change-sensitive manner. As such, the approach of structured professional judgment is in current use and is considered to be the most advanced approach to threat assessment of target-based violence prevention (Douglas, 2009; Guy et al., 2012; Meloy et al., 2012; Simon \& Tardiff, 2008; Skeem \& Mulvey, 2001).

\section{Theme 3: Leakage, Risk Factors and Warning Behaviors}

Overview. In general, TATs function best when comprised of multidisciplinary members with proper training and experience. They should be trained to identify, assess, and manage situations, key warning behaviors, and risk factors to mitigate targeted violence. TATs must also understand the statistical significance of target-based behavioral indicators, the precipitating conditions leading to targeted violence, the psychological processes of perpetrators, and relevant prevention interventions to targeted violence. Understanding the significance of target-based violent behaviors, the precipitating conditions and psychological processes of perpetrators, and relevant prevention interventions for targeted violence is also essential groundwork for TATs. 2

Leakage. The concept of leakage as it relates to threat assessment was coined by O'Toole (2000) in her study of the school shooter. Leakage is a warning behavior that consists of revealing clues through communication to a third party through various methods such as: e-mails, letters, tweets, social media (e.g., Facebook, YouTube), drawings, stories, poems, etc. (Meloy \& O'Toole, 2011). This warning behavior is considered the most important clue preceding an adolescent's violent act (Twemlow, Fonagy, Sacco, O'Toole, Vernberg, \& Jellinek, 2002). In the Secret Service Exception Case Study Project, researchers examined the situations of $(N=83)$ subjects planning or attempting assassinations of public figures and found that $63 \%$ had various forms of leakage (Fein \& Vossekuil, 1998, 1999).

Leakage is considered to be very common in school shootings (Augustyniak, 2005; Bondü et al., 2011; Meloy \& O'Toole, 2011). In a study conducted by the U.S. Secret Service on school shooters, at least one individual knew the shooter was thinking about or planning the shooting in $81 \%$ of the cases and in $59 \%$ of the cases more than one person know (Vossekuil, Reddy, Fein, Borum, \& Modzelski, 2000). These findings suggest that leakage identification plays a primary role in determining how to prevent violent acts (Augustyniak, 2005; Bondü et al., 2011; Meloy \& O'Toole, 2011; O'Toole \& Häkkänen-Nyholm, 2012).

The problem is that persons who witness leakage of the perpetrator's plan to commit violence usually remain silent and fail to notify an authority who could intervene and prevent the violence from happening (Augustyniak, 2005; Keller, Hughes, \& Hertz, 2011; Pollard, Noland, \& Deisinger, 2012). Because leakage 
presents an opportunity to identify and intercept violent acts, a prime aspect of most TATs is to create a social context in which reporting leakage is encouraged, as well as a community infrastructure that is prepared to act on reports in order to prevent school violence (Cornell, 2011; Scalora et al., 2010). This process requires educating students and school employees on how to identify leakage and empowering them to report leakage to school officials or TAT members.

Risk factors and warning behaviors. According to Meloy and O'Toole (2011), warning behaviors associated with leakage include research, planning, preparation, or implementation of an attack (Calhoun \& Weston, 2003), and behaviors that indicate a pathological preoccupation with violence that is connected to a particular person (Mullen et al., 2009). Leakage appears to be one of the most readily available initial warning behaviors of shooters and can thus be utilized to signal a need to investigate and assess the person in question. Through conducting a thorough assessment, TAT members can develop a comprehensive understanding of the person's warning behaviors and life dynamics in order to make an efficient decision (Van Brunt, 2012).

Meloy et al. (2012) stated that "Warning behaviors constitute particularly toxic changes in patterns of behavior which require an operational response" ( $p$. 6). Another important consideration is the imminence of the attack, which pertains to "an increasing probability of the act occurring within a specific time-frame" (Meloy et al., 2012, p. 6). Meloy et al. also emphasized that risk factors and the degree of risk involved in a potential threat of violence is a dynamic and constantly changing process that must be continually tracked until the threat is fully diminished.

Key investigative questions. A crucial aspect of threat assessment for target-based violence is utilizing key questions to determine whether there is evidence to suggest movement toward violent action (Reddy et al., 2001). Specific questions help to discern the presence and magnitude of risk factors and warning behaviors that are vital in determining the assessment of the threat for violence. Borum et al. (2010) detailed the core questions that enable a fuller understanding of the threat. A representative question from Borum et al. includes, "Does the student see violence as an acceptable - or desirable - or the only-way to solve problems?" (p. 32).

Investigative questions attempt to collect any evidence of an interest or intent in committing violence, along with motivations, psychological or emotional state, and ideas about violence that would suggest the presence of a threat. The Salem-Keizer system for assessing threats of violence in schools utilizes additional questions to probe for a fuller picture of the situation (Van Dreal, 2011). A few of the areas these questions explore are: the involvement of other students or persons in the threat plan; the level of concern of other students and teachers regarding 
the subject of concern; the main source of information about the subject of concern; and the degree of development and specificity in the subject's research, plan, and/or communication of the threat (Van Dreal, 2011). After combining information from all these perspectives regarding the presence of a threat, a better understanding of the degree of the threat, as well as modes of intervention, become possible.

Contemporary approaches to threat assessment of target-based violence, especially in IHEs, utilize a highly structured professional approach that involves an elaborate protocol for utilizing investigative questions to determine the level of risk in any given threat (Randazzo \& Cameron, 2012). Because this field of inquiry is relatively new and not yet fully understood, its ability to work practically towards the identification of threat and prevention of violence is in an early developmental stage. Yet it is imperative that research, planning, and the development of strategies to assess threats and prevent violence in schools continue to evolve.

\section{Theme 4: Defining the Problem of School-Targeted Violence Related To IHEs}

Overiew. To develop a comprehensive awareness of the problem, it is important to look at the diverse aspects of school shootings, including: the statistical data regarding subjects and victims, the environmental and social contexts, the motivations of shooters, and the prevention and violence management strategies that TATs can utilize (Randazzo \& Cameron, 2012). A distinction can also be made between threat assessment strategies for K-12 and IHEs (Sulkowski \& Lazarus, 2011).

Statistical data. In 2010, the FBI released a report that analyzed violent and lethal incidents - including school shootings - in IHEs between 1900 and 2008; the report focused on defining the most important data regarding subjects and victims involved, as well as the prevalence of these violent acts (Drysdale, Modzeleski, \& Simons, 2010). Drysdale et al. reported that "Across all 272 incidents, the subjects caused 281 deaths and injured 247 individuals. Of the deaths, at least 190 were students and at least 72 were employees. Of the injured, at least 144 were students and at least 35 were employees. Not included in these numbers are the subjects themselves who were injured or killed either during or following the incident" (p.17). This FBI document went on to report a number of variables associated with these targeted attacks.

The FBI report also found that $84 \%$ of the violent incidents took place at 4 -year universities, almost all were perpetrated by one person, and $94 \%$ of the shooters were male (Drysdale et al., 2010). Additionally, $60 \%$ of the shooters were students at the IHE (either current or former); $20 \%$ of the shooters were in a 
relationship with a student or employee at the IHE; and $11 \%$ were employees (either current or former) of the IHE. Lastly, the FBI report found evidence of leakage in many of the violent incidents: in $13 \%$ of the cases, subjects threatened victims verbally or in writing; $19 \%$ of subjects stalked or harassed victims; $10 \%$ of subjects engaged in violent behaviors upon victims; and in $31 \%$ of the cases, outside observers of the subject were aware of suspicious or threatening behaviors of concern.

The FBI report also concluded that in over $75 \%$ of the cases, a specific target was in mind, and the violence was usually enacted as retribution in the context of a relationship (Drysdale et al., 2010). Along these lines, it is vital for TATs to better understand the psychological and emotional stressors and motivations that lead to incidents of violence and school shootings.

The distinction between K-12 and IHE shootings. Providing threat assessment and prevention of target-based violence at IHEs involves an understanding of the unique social and environmental conditions present at these institutions. Citing the work of Boynton (2003), Sulkowski and Lazarus (2011) stated, "college campuses are prime locations for violent perpetrators to stage devastating multiple victim attacks due to their dense populations, relatively low police presence, and open and welcoming nature" (p. 338). Further, IHEs typically feature larger diverse campuses than $\mathrm{K}-12$, on which strict monitoring - such as metal detectors and physical access control - is not appropriate (Fox \& Savage, 2009). The open college environment has also become a place for community members to congregate, attend functions, and explore resources (Bolante \& Dykeman, 2015).

Due to their adult age status, students at IHEs maintain greater expectations of social freedoms and expression than do K-12 students. Relationship dynamics between students at IHEs also feature a greater degree of physical and sexual intimacy, which could lead to riskier behaviors and/or more profound experiences of distress - and the likelihood of acting out - with disturbances in the relationships. School officials at IHEs cannot as easily report student problems to parents or elicit parental support with correcting poor behaviors. In other words, both students at IHEs and the social environment of IHEs are more like small-scale communities of adults than are $\mathrm{K}-12$ schools. This fact produces greater challenges for managing threats of violence at IHEs.

\section{Discussion}

This review of literature has demonstrated that the optimal approach to identifying, managing, and preventing target-based threats of violence at IHEs is not a simple matter of using "cookie cutter" reactions. The optimal approach to 
threat management is proactive and not reactive, and requires a comprehensive review of the subject's psychosocial and behavioral dynamics. Effective threat assessment also involves an individualistic approach of structured professional judgment to each case, not a generalized response based on actuarial algorithms. An effective IHE TAT will empower team members to assess threats innovatively as dynamic situations that are constantly changing.

Most importantly, the problem and prevention of target-based violence at IHEs must be viewed as the responsibility of the community. IHE officials must be aware of students who exhibit warning behaviors that signal profound psychosocial distress and respond with appropriate support strategies to stabilize and mitigate potential violence. Counselor training issues are plentiful and will need to be addressed in future research and training. Issues such as information sharing, confidentiality issues, ethical dilemmas, and skill-specific topics, such as emphasis on coping skills, suicide prevention techniques, recognizing and addressing homicidal ideation, and recognizing pre-incident indicators, must be addressed. Threat assessment, as applied to the counseling profession, is different from mental health, traditional counseling, and behavioral intervention practices and warrants specialized training and understanding of the role of counselors within a TAT (Van

Dreal, Rainwater, \& Okada, 2011). Although the field of threat assessment/management is in an early stage of development, it is very important to examine the various training needs for professionals in the counseling field (Bolante \& Dykeman, 2015; Perloe \& Pollard, 2016).

\section{References}

Albrecht, S. (2010, February). Campus safety: Threat assessment teams strive to prevent or manage violence at schools and workplaces. FBI Law Enforcement Bulletin, 79(2), 15-24. Retrieved from

http://leb.fbi.gov/2010/february/leb-february-2010

Augustyniak, K. M. (2005). Integration of Federal Bureau of Investigation and United States Secret Service/Department of Education threat assessment models into a conceptual framework for prevention of school violence.

Journal of School Violence, 4(2), 29-46. http://dx.doi.org/10.1300/J202v04n02_03

Bernes, K. B., \& Bardick, A. D. (2007). Conducting adolescent violence risk assessments: A framework for 419 school counselors. Professional School Counseling, 10(4), 419-427. http://dx.doi.org/10.5330/prsc.10.4.e43404402j07480u

Bolante, R., \& Dykeman, C. (2015). Threat assessment in community colleges. Journal of Threat Assessment and Management, 2, 23-32. 
http://dx.doi.org/10.1037/

$\operatorname{tam} 0000033$

Bondü, R., Cornell, D. G., \& Scheithauer, H. (2011). Student homicidal violence in schools: An international problem. New Directions For Youth

Development, 129, 13-30. doi:10.1002/yd.384

Borum, R., Cornell, D. G., Modzeleski, W., \& Jimerson, S. R. (2010). What can be done about school shootings? A review of the evidence. Educational Researcher, 39(1), 27-37. doi:10.3102/0013189X09357620

Boynton, A. (2003). Securing college campuses in the face of terrorism. Campus Law Enforcement Journal, 33, 15-17. Retrieved from http://www.ncjrs.gov/App/ publications/abstract.aspx?ID $=203626$

Calhoun, F., \& Weston, S. (2003). Contemporary threat management: A practical guide for identifying, assessing, and managing individuals of violent intent. San Diego, CA: Specialized Training Services.

Cornell, D. (2010). Threat assessment in college settings. Change: The Magazine of Higher Learning, 42(1), 8-15. Retrieved from http://www.changemag.org/ Archives/Back\%20Issues/January-February\%202010/full-threatassessment.html

Cornell, D. (2011). A developmental perspective on the Virginia student threat assessment guidelines. New Directions for Youth Development, 129, 4359. doi:10.1002/yd.386

Cornell, D., \& Allen, K. (2011). Development, evaluation, and future directions of the Virginia student threat assessment guidelines. Journal of School Violence, 10(1), 88-106.

Cornell, D., Sheras, P., Gregory, A., \& Fan, X. (2009). A retrospective study of school safety conditions in high schools using the Virginia threat assessment guidelines versus alternative approaches. School Psychology Quarterly, 24(2), 119-129.

Douglas, K. S. (2009). Expert opinion. Assessing risk for violence using structured professional judgment. American Psychology-Law Society News, 29, 12-15.

Drysdale, D., Modzeleski, W., \& Simons, A. (2010). Campus attacks: Targeted violence affecting institutions of higher education. Washington, DC: U.S. Secret Service, U.S. Department of Education, and Federal Bureau of Investigation. 
Fein, R., \& Vossekuil, B. (1998). Preventing attacks on public officials and public figures: A Secret Service perspective. In J. R. Meloy (Ed.), The psychology of stalking: Clinical and forensic perspectives (pp. 175-191). San Diego, CA: Academic Press.

Fein, R. A., \& Vossekuil, B. (1999). Assassination in the United States: An operational study of recent assassins, attackers, and near-lethal approachers. Journal of Forensic Science, 44, 321-333. https://doi.org/10.1520/JFS14457J

Fox, J., \& Savage, J. (2009). Mass murder goes to college: An examination of changes on college campuses following Virginia Tech. American Behavioral Scientist, 52, 1465-1485. doi:10.1177/0002764209332558

Guy, L. S., Packer, I. K., \& Warnken, W. (2012). Assessing risk of violence using structured professional judgment guidelines. Journal of Forensic Psychology Practice, 12, 270-283. http//dx.doi.org/10.1080/15228932.2012.674471

Higgins, J. P. T., \& Green, S. (2011). Cochrane Handbook for Systematic Reviews of Interventions, Version 5.1.0. London: The Cochrane Collaboration. Retrieved from: http://training.cochrane.org/handbook

Indiana Department of Education, Safety Academy. (2017). Threat assessment template. Indianapolis, IN: Author. Retrieved from http://www.doe.in.gov/sites/ default/files/safety/template.docx

Keller, E. W., Hughes, S., \& Hertz, G. (2011). A model for assessment and mitigation of threats on the college campus. Journal of Educational Administration, 49, 76-94. http://dx.doi.org/10.1108/09578231111102072

Meloy, J. R., \& Genzman, J. (2016). The clinical threat assessment of the loneactor terrorist. Psychiatric Clinics of North America, 39(4), 649-662. http://dx.doi.org/10.1016/j.psc.2016.07.004

Meloy, J., Hoffmann, J., Guldimann, A., \& James, D. (2012). The role of warning behaviors in threat assessment: an exploration and suggested typology. Behavioral Sciences and the Law, 30(3), 256-279. doi:10.1002/bsl.999

Meloy, J., \& O'Toole, M. E. (2011). The concept of leakage in threat assessment. Behavioral Sciences and the Law, 29, 513-527. doi:10.1002/bsl.986

Moher, D., Liberati, A., Tetzlaff, J., Altman, D. G., \& PRISMA Group. (2009). Preferred reporting items for systematic reviews and meta-analyses: The PRISMA statement. Annals of Internal Medicine, 151(4), 264-269. doi:10.7326/0003-4819-151-4-200908180-00135 
Mullen, P., James, D., Meloy, J. R., Pathé, M., Farnham, F., Preston, L., Darnley, B., \& Berman, J. (2009). The fixated and the pursuit of public figures. Journal of Forensic Psychiatry and Psychology, 20, 33-47.

http://dx.doi.org/10.1080/ 14789940802197074

Nolan, J. J., Randazzo, M. R., \& Deisinger, G. (2011). Campus threat assessment and management teams: What risk managers need to know now.

University Risk Management and Insurance Association Journal, 15, 105122 .

Ottawa Hospital Research Institute. (2009). Five top medical journals publish research guidelines developed in Ottawa. Ottawa, ON: Author. Retrieved from http://www.ohri.ca/newsroom/newsstory.asp?ID=185

O'Toole, M. E. (2000). The school shooter: A threat assessment perspective. Quantico, VA: National Center for the Analysis of Violent Crime, Federal Bureau of Investigation. Retrieved from http://files.eric.ed.gov/fulltext/ED446352.pdf

O’Toole, M. E., \& Häkkänen-Nyholm, H. (2012). Psychopathy and violent crime. In H. Häkkänen-Nyholm, \& J. Nyholm (Eds.), Psychopathy and law: A practitioner's guide (pp. 139-175). Walden, MA: Wiley.

Perloe, A., \& Pollard, J. (2016). University counseling center's role in campus threat assessment and management. Journal of Threat Assessment and Management, 3, 1-20. http://dx.doi.org/10.1037/tam0000051

Pollack, W. S., Modzeleski, W., \& Rooney, G. (2008). Prior knowledge of potential school-based violence: Information students learn may prevent a targeted attack. Washington, DC: U.S. Secret Service and U.S. Department of Education.

Pollard, J., Noland, J., \& Deisinger, E. (2012). The practice of campus-based threat assessment: An overview. Journal of College Student Psychotherapy, 26, 263-276. http://dx.doi.org/10.1080/87568225.2012.711142

PRISMA. (2017). PRISMA flow diagram. Ottawa, ONT: Author. Retrieved from http://www.prisma-statement.org/PRISMAStatement/FlowDiagram.aspx

Randazzo, J., \& Cameron, K. (2012). From presidential protection to campus security: A brief history of threat assessment in North American schools and colleges. Journal of College Student Psychotherapy, 26, 277-290. http://dx.doi.org/ $10.1080 / 87568225.2012 .711146$ 
Reddy, M., Borum, R., Berglund, J., Vossekuil, B., Fein, R., \& Modzeleski, W. (2001). Evaluating risk for targeted violence in schools: Comparing risk assessment, threat assessment, and other approaches. Psychology in the Schools, 38(2), 157-172.

Scalora, M., Simons, A., \& VanSlyke, S. (2010). Campus safety: Assessing and managing threat. FBI Law Enforcement Bulletin. Retrieved from https://leb.fbi.gov/2010/february/campus-safety-assessing-and-managingthreats

Simon, R. I., \& Tardiff, K. (2008). Textbook of violence assessment and management. Arlington, VA: American Psychiatric Association Publishing.

Skeem, J. L., \& Mulvey, E. P. (2001). Psychopathy and community violence among civil psychiatric patients: Results from the MacArthur Violence Risk Assessment Study. Journal of Consulting and Clinical Psychology, 69, 358-374. http://dx.doi.org/ 10.1037/0022-006X.69.3.358

Sulkowski, M. L., \& Lazarus, P. J. (2011). Contemporary responses to violent attacks on college campuses. Journal of School Violence, 10, 338-354. http://dx.doi.org/ 10.1080/15388220.2011.602601

Tuttle, H. (2015). Preparing for an active shooter incident. Risk Management, 62(9), 4. Retrieved from http://www.rmmagazine.com/2015/11/02/preparing-for-an-activeshooter-incident/

Twemlow, S. W., Fonagy, P., Sacco, F. C., O'Toole, M. E., Vernberg, E., \& Jellinek, M. S. (2002). Premeditated mass shootings in schools: Threat assessment. Journal of the American Academy of Child 8 Adolescent Psychiatry, 41, 475-477. http://dx.doi.org/10.1097/00004583-20020400000021

University of Arkansas at Little Rock Public Safety Department. (2017a). Threat assessment team. Little Rock: University of Arkansas at Little Rock. Retrieved from http://ualr.edu/safety/home/campus-safetylinks/emergency-management-plan/threat-assessment-team/

University of Arkansas at Little Rock Public Safety Department. (2017b). Types of threats. Little Rock: University of Arkansas at Little Rock. Retrieved from http://ualr.edu/safety/home/campus-safety-links/emergencymanagement-plan/threat-assessment-team/types-of-threats/ 
US Department of Education. (2003). Identifying and implementing educational practices supported by rigorous evidence: A user friendly guide. Washington, DC: Author. Retrieved from https://ies.ed.gov/ncee/pubs/evidence_based/evidence_based.asp

US Department of Education. (2017). What is a threat assessment team? Washington, DC: Author. Retrieved from http://familypolicy.ed.gov/content/what-threat-assessment-team

Van Brunt, B. (2012). Ending campus violence: New approaches to prevention. New York, NY: Routledge.

Van Dreal, J. (2011). Assessing student threats: A handbook for implementing the Salem-Keizer system. Lanham, MD: Rowman \& Littlefield.

Van Dreal, J., Cunningham, M., \& Nishioka, V. (2005). Mid-Valley student threat assessment system: Making schools safer through a multi-agency collaboration. Paper presented at Persistently Safe Schools 2005: The National Conference of the Hamilton Fish Institute on School and Community, Paper \#9505, 249-258.

Van Dreal, J., Rainwater, A., \& Okada, D. (2011). Level 2 investigation team. In J. Van Dreal (Ed.), Assessing student threats: A handbook for implementing the Salem-Keizer system (pp. 123-138). Lanham, MD: Rowman \& Littlefield.

Virginia Department of Criminal Justice Services. (2014). Virginia Center for School and Campus Safety. Richmond, VA: Author. Retrieved from https://www.dcjs.virginia.gov/virginia-center-school-and-campus-safety Vossekuil, B., Fein, R. A., Reddy, M., Borum, R., \& Modzeleski, W. (2004). The final report and findings of the Safe School Initiative: Implications for the prevention of school attacks in the United States. Washington, DC: U.S. Secret Service and U.S. Department of Education.

Vossekuil, B., Reddy, M., Fein, R., Borum, R., \& Modzeleski, M. (2000). USSS safe school initiative: An interim report on the prevention of targeted violence in schools. Washington, DC: U.S. Secret Service, National Threat Assessment Center.

\section{Author Biographies}

Bolante, Rebecca, Threat Management Resources Director, Chemeketa Community College. Rebecca Bolante, PhD, CRC, CTM- Director of Threat Management Resources, Chemeketa Community College, Salem, Oregon. Email: rebecca.bolante@bolante.net 
Rebecca Bolante, $\mathrm{PhD}$, is the Director of Threat Management Resources, Chemeketa Community College located in Salem, Oregon. Bolante provides training and teaches online courses in threat assessment and trauma response. Bolante provides consultation for various institutions, agencies, and entities as we work together to mitigate targeted violence. She is a trainer and consultant for the college's threat assessment team and the trauma response team. Dr. Bolante has developed standardized processes for the psychological response after a disaster. Recently she provided her expertise and assistance after the Umpqua Community College incident. This included evaluating the various needs, setting up support rooms, and coordinating resources for both civilians and the EMS community. As a Certified Rehabilitation Counselor, Bolante has gained insight from her more than 25 years of work within the field of disabilities. Dr. Bolante is a Certified Threat Manager through the Association of Threat Assessment Professionals. Rebecca Bolante is also active with the ASIS International as she is currently the 2nd Vice Chair of the School Safety \& Security Council.

Dykeman, Cass, Associate Professor of Counseling, Counseling Academic Unit, College of Education, Oregon State University, Corvallis, Oregon. Email: dykemanc@oregonstate.edu

Cass Dykeman, $\mathrm{PhD}$, is an Associate Professor of Counseling at Oregon State University. At OSU, he has served as served as a unit lead, department chair, and associate dean for research. In addition, he served as the director of the Career Development Taxonomic Research Project of the National Center for Research in Career and Technical Education. Dr. Dykeman is a former high school and elementary school counselor in Seattle, WA. He has served as the principal investigator for two federal grants and is the author of numerous books, book chapters, and scholarly articles in the area of counseling. 